\title{
ZUR LAUT- UND FORMENLFHRE DER ALTNORDISCHEN SPRACHEN.
}

I. 3. pl. conj. isl. $-i$ : a schw. -in (biojpi: biupin) und nom., acc. pl. der neutralen $n$-stämme isl. $-u$ : aschw. -on

(augu : oghon).

Die schwestersprachen isl. und aschw. gehen bekanntlich in diesen formen auseinander. Die aschw. oghon, oron entsprechen den isl. augu, eyru, hiortu etc.; im isl. geht die 3. pers. pl. praes. und praet. conj. immer auf -i (bióbi, fóri), im normalen aschw. aber auf -in (biubin, forin) aus. Während der älteren aschw. periode begegnet nämlich die endung $-e(-i)$ hauptsächlich nur in solchen schriften, welche auch sonst dialektische altnorwegische (isl.) zulge zeigen (in dem dialekte von Vestergötland; vgl. Rydqvist: Svenska språkets lagar I, 345 f., Kock: Studier öfver fornsvensk ljudlära II, 509). Keine befriedigende erklärung dieser differenz der altnord. sprachen durfte bisher gefunden sein.

Läffler nimmt (Tidskrift for Filologi N. R. V, 77) an, dass die aschw. formen oghon, biupin lautgesetzlich aus den got. augona, biudaina entwickelt sind, dass aber die isl. augu, biópi das lautgesetzliche $-n$ analogisch eingebusst haben. Nach seiner meinung soll *biópin unter einfluss der 3. pl. ind. bióba zu biópi umgestaltet worden sein, und *augun durch partiellen anschluss an nom. und acc. pl. der masculinen und femininen $n$-stämme (*boga, *tungu) die entstehung der neuen form augu veranlasst haben (vgl. auch Tamm: Om tyska ändelser i svenskan s. 37 note). Die von Laffler postulierte analogieeinwirkung um biópi zu erklären, scheint mir doch allzu compliciert um wahrscheinlich zu sein. Die endungen in biópa und biópin sind so unäbnlich, dass man keine partielle ein- 
wirkung der formen auf einander annehmen kann. Wenn *biópin von biópa beeinflusst werden soll, so erwartet man, dass der endvocal $-a$ aus dieser in jene form ubertragen wird, aber nicht dass nur $-n$ in *biópin fällt. Die erklärung Läfflers von isl. augu wird hierdurch auch verdächtig, da aschw. oghon sich $\mathrm{zu}$ isl. augu verhält wie aschw. biupin $\mathrm{zu}$ isl. biópi.

Mit recht hat also Noreen in seiner verdienstlichen abhandlung 'Geschichte der nordischen sprachen' (in Pauls Grundriss der germ. philologie I) die erklärung Läff lers nicht acceptiert. Um isl. augu : aschw. oghon, isl. bióbi : aschw. biupin zu deuten wird er aber genötigt einen ganz verschiedenen ursprung der isl. und der aschw. formen anzunehmen (ib. § 175, 7; $\S 232,3)$. Im aschw. oghon sieht er naturlich das got. augona, aber 'daneben steht (meint er) die dem ahd. -un entsprechende endung $-u$, welche (ursprünglich wol duale form) nur den westnordischen geläufig ist, z. b. aisl. hiú, augo, oyro'. Und obgleich aschw. biupin dem got. biudaina entspricht, soll nach ihm isl. biópi auf ein construiertes got. *biudain zurïckzufuhren sein (vgl. auch Kluge in Pauls Grundriss I, 382).

Diese annahmen scheinen mir sehr kuhn. Es gibt kein got. *biudain; in keiner anderen germ. sprache muss die 3. pers. conj. direkt auf ein germ. *teuðain(d) zurückgefuhrt werden (Kluge a. a. o.), und es ist selbstverständlich dass man, wenn eine andere erklärung möglich ist, oghon und augu, biupin und biópi in zwei so nahverwanten dialekten wie dem aschw. und dem isl. nicht scheiden darf. Hierzu knmmt noch dass, wenn oghon und augu, biupin und biófi von verschiedenen grundformen ausgegangen, es ein sonderbarer zufall wäre, dass in beiden fällen das aschw. die formen mit $-n$, das isl. aber die formen olıe $-n$ gewählt hätte.

Meiner meinung nach ist sowol isl. augu als aschw. oghon auf eine ältere form, die mit got. augōna identisch war, zurluckzufuhren, und sowol isl. bióf,i als aschw. biupin sind aus einem älteren (= got.) biudaina entwickelt, aber der verlust des - $n$ im isl. ist nicht, wie Läffler annahm, durch analogie-einwirkung zu erklären; er ist lautgesetzlich. Es ist wol der umstand, dass das isl. z. b. im acc. sg. masc. gópan (got. godana) das - $n$ erhalten, welcher die annahme veranlasst hat, dass isl. augu, biópi icht lautgesetzlich aus (got.) augōna, biudaina ent- 
wickelt sein können. Wenn aber die gesetze fur den verlust des auslautenden - $n$ der endsilben richtig formuliert werden, machen goban etc. keine schwierigkeit.

Man muss sich nämlich erinnern, dass das auslautende - $n$ der nordischen sprachen in verschiedenen stellungen während sehr verschiedener perioden eingeblisst worden ist, und dass z. b. einige neuschwedische dialekte $\cdot n$ in solchen stellungen verloren haben, wo andere dialekte es noch aufrecht erhalten. Während schon im gemeinnord. das auslautende $-n$ des got. wegfiel (z. b. in inf. bita got. beitan; in acc. lofa got. lofan), so zeigen das isl. und das normale aschw. noch $-n$ in útan, saman etc. (got. utana, samana etc.). Aber in diesen partikeln ist das - $n$ später in gewissen dialekten abgefallen, so dass man utta, samma etc. bekommen (spuren solcher dialektformen begegnen schon im aschw.; siehe Kock: Arkiv N. F. II, 32 note), obgleich utan, saman etc. in mehreren gegenden das $-n$ noch belialten.

Unter diesen verhältnissen befremdet es nicht, dass schon in den verschiedenen altnord. dialekten verschiedene lautgesetze fur das auslautende $-n$ zur geltung gekommen sind. Nachdem die gemeinnord. formen bita, lófa etc. sich entwickelt, und nachdem in dreisilbigen wörtern godana, augona, biudaina etc. der vocal der ultima eingebtisst worden war, so wurde während einer etwas späteren periode im isl. (aber nicht im aschw.) folgendes lautgesetz durchgefuhrt: auslautendes $-n$ fällt in relativ unbetonter silbe weg, wenn der vorhergehende vocal lang (aber nicht, wenn er kurz) ist. Ob der vocal zu dieser zeit lang oder kurz war, entscheidet das got. Got. hat langen vocal (resp. diphthong) in penultima von augōna, foreina (3. pl. praet.), biudaina; isl. hat deshalb das auslautende $-n$ in augu, fóri, biópi (aus *augōn, *fō rīn, *biuð̄enn) eingeblisst. Weiter unten wird dargetan werden, dass auch bábi (nom., acc. von bábir) in derselben weise aus bāeiñ (agutn. bepin) entstanden ist. Der vocal der penultima ist aber kurz in got. godana etc., utana, innana etc., und das isl. hat deshalb $-n$ in gópan etc., útan, innan etc. erhalten.

Es ist lautphysiologisch sehr begreiflich, dass der auslautende consonant der unbetonten silbe leichter verklingt, wenn der vorhergehende vocal lang, als wenn er kurz ist. 
Göpann und *augōn wurden gleich accentuiert, und die ultima beider wörter war relativ unbetont. Der geringe exspirationsdruck der ultima nahm gegen das ende der silbe ab, und der $n$-laut wurde also am schwächsten ausgesprochen. Bei gleichem exspirationsdruck auf ultima von $g \bar{o} p \not \bar{l} n$ und *augōn resultierte hieraus, dass der $n$-laut der längeren silbe $-\bar{o} n$ eine schwächere aussprache bekam und leichter wegfiel als der $n$-laut der kurzeren silbe -ăn.

Meine ansicht wird von pl. hion mit erbaltenem - $n$ (neben hiú) bestätigt. Nachdem dieser urspr. n-stamm den hauptton (fortis) schon früh auf ultima versetzt hatte und einsilbig geworden war, konnte er das auslautende $-n$ nicht mehr einbussen.

Aus dieser darstellung ergibt sich auch, dass isl. die langen endrocale in wörtern mit langer wurzelsilbe relativ lange erhalten hat, wcuigstens wenn sie einen endvocal (in der dritten silbe) fruher eingeblisst haben, und dies ist nicht befremdend, da die altnord. sprachen in sehr später zeit, und zwar noch lange nachdem das $-n$ in isl. augu, biópi etc. weggefallen war, in wörtern mit kurzer wurzelsilbe die urspr. langen endrocale erhalten haben (Arkiv IV, $87 \mathrm{ff}$., Beiträge XIV, 70 note).

Unter diesen umständen wurde vielleicht jemand fragen, warum nicht das auslautende $-n$ in z. b. gōpăn und falān (acc. sg. masc. von gōjor und $f(a l r)$, je nachdem der vocal der ultima kurz oder lang war; in verschiedener weise behandelt worden ist, so dass man gōbăn aber *fălā bekommen hätte. Die antwort liegt aber nahe. Es gab zwei umstände, von welchen jeder für sich hinlänglich war um den $n$-laut in falan etc. zu conservieren. Die länge der ultima hing in diesen wörtern (im gegensatz zu den oben besprochenen *augōn etc.) von der betonung ab: ultima war nicht nur lang, sondern sie wurde mit einem starken nebenton (levis, siehe Kock, Arkiv a. a. o., Studier öfver fsv. ljudlära II, $340 \mathrm{ff}$., und vgl. jetzt auch Noreen in Pauls Grundriss §52, II, 3) ausgesprochen. $\mathrm{Da}$ also die ultima von falan einen stärkeren exspiratorischen accent als die ultima von *augōn trug, so erwartet man nicht, dass das auslautende $-n$ in beiden wörtern gleich behandelt werde. Wenn aber auch dies nicht der fall gewesen 
wäre, so würde doch ohne zweifel falan den $n$-laut erhalten haben, und zwar durch anschluss an die wörter mit kurzem endvocal, d. h. an wörter mit langer wurzelsilbe und an mehr als zweisilbige wörter. Diese wortkategorien sind nämlich vielmal zahlreicher als die zweisilbigen wörter mit kurzer wurzelsilbe.

Im neuschw. haben ögon, öron eben so wie die neudän. oien, oren das auslautende $-n$ erhalten; die 3 . pers. pl. conj. (bjude, fore etc.) hat aber im neuschw. die form des ganzen sing. und die der 1.pers. pl. angenommen, und schon im altdän. ist der conj. indeclinabel geworden (biudhce etc., vgl. Noreen in Pauls Grundriss $\S 15,2)$, d. h. die alte ostnord. endung -in ist durch anschluss an die formen mit $i$ umgebildet worden, eben so wie diese umbildung im schw. später eingetreten ist.

Aus obiger darstellung ergibt sich natürlich auch, dass der isl. nom. acc. pl. neutr. gópu (in der schwachen form) sich lautgesetzlich aus got. godōna entwickelt baben kann. Da aber im aschw. der pl. der schwachen adj. in allen formen auf -o (gopo) ausgeht, und also hier ubertragungen nach einem grossen massstab stattgefunden haben, so befremdet es nicht, dass auch nom. und acc. pl. neutr. *gopon (durch anschluss an fem. gopo) gopo geworden, und im isl. kann nom. acc. pl. neutr. góbu naturlich auch in dieser weise erklärt werden (vgl. Läffler, Tidskrift for Filologi N. R. V, 75).

II. Einige pronominalformen und zahlwörter.

1. Isl. bábi, ostnord.bepin, bape. Diese oben (s. 246) genannten formen werde ich hier etwas ausfuhrlicher besprechen. Bekanntlich ist bábir eine zusammenschmelzung von zwei wörtern: bai und pai 'beide die' (Sievers, Beiträge X, 495; Meringer, KZ. XXVIII, 236), und die ursache der entwickelung von $b a i$ in $b \bar{a}$ ist darin ist zu suchen, dass der hauptton (fortis) fruher auf dem zweiten juxtapositionsglied rubte (Falk, Arkiv N. F. II, 114 note).

Es dürfte aber nicht befriedigend erklärt worden sein, weder woher das $-n$ in nom., acc. pl. neutr. agutn. bepin, aschw. babi(n) stammt, noch warum diese form umgelautet ist (isl.bépi, agutn. bepin). Jene frage sucht Läffler ('Tidskrift f. Filologi N. R. 
V, 76 note 5) so zu beantworten, dass babe(n), (baepin) das auslautende $-n$ von den wörtern sighon, hion übernommen haben, weil die zusammenstellungen $b a b e(n)$ gghon, babe(n) hion geläufig waren, und er weist noch auf cengin, hvarghin als factoren, die zur einfuhrung des $-n$ in bapin beigetragen hätten, hin.

Es wäre doch sehr sonderbar, wenn eine so gewöhnliche pronominalform wie babin das auslautende $n$ wesentlich durch verbindung mit ein paar auf $n$ auslautenden substantiven bekommen hätte, und ich kann mich deshalb dieser meinung nicht anschliessen.

Es wäre naturlich erwunscht, dass man eine gemeinsame erklärung sowol für den zusatz von $n$ in bepin als fur dessen umlaut finden könnte, und dies ist meiner ansicht nach möglich. Ich sehe nämlich in bepin eine zusammenrlickung von $b \bar{a} p i+h i n$. Ebenso wie báfir aus bai pai 'beide die' entstanden ist, so bat man, nachdem der ursprung des wortes vergessen worden, und nachdem $b \bar{a} p i r$, neutr. $b \bar{a} p i$ die flexion von pessi (Meringer a. a. o.) angenommen hatte, noch einmal neutr. $b \bar{a} p i$ mit hin (neutr. pl. von hinn 'jener', got. himma, hina, hita 'dieser') vereinigt. Die bedeutung von $b \bar{a} p i$ hin war 'beide die' ebenso wie die ursprungliche bedeutung von bai bai dieselbe gewesen war. Inlautendes $h$ schwand wie gewöhnlich, und von $b \bar{a} b i-(h)$ in wurde $b \bar{a} p \bar{p} n$. Durch den jungeren $i$-umlaut (vgl. Kock, Arkiv IV, 141 ff., Beiträge XIV, 53 ff.) bekam man $b \bar{x} \overline{b i n}$, welche form im agutn. bepin begegnet. Ebenso wie aber das auslautende $-n$ nach einem langen ultimavocal in isl. * fórīn, *augōn etc. wegfiel, so fiel es auch in $b \bar{a} \bar{b} \bar{\imath} n$, so dass man bépi bekam. Neben agutn. bepin hat man auch agutn. $b a b i$, aschw. babe, und dies ist das urspr. $b \bar{a} b i$ ohne hinzugefugtes hin. Nun versteht man, warum agutn. bejin mit $-n$ umgelautet, agutn. $b a b i$ aber ohne $-n$ unumgelautet ist. Das aschw. babin ist eine contaminationsform von babe und baepin. Den isl. bábir (nom. pl. masc.) und bébi (neutr.) entsprechen die agutn. bepir und bepin, und im agutn. ist also auch nom. masc. umgelautet. Der umlaut ist wol aus neutr. bepin eingedrungen, und es ist kaum wabrscheinlich, dass agutn. bepir eine zusammenschmelzung von $b \bar{a} b i-\bar{\imath} r \quad(\bar{\imath} r=$ got. nom. pl. masc. $e i s)$, ebenso wie bepin von baji+(h)in, wäre. 
Diese erklärung von bepin, bapin wird dadurch bestätigt, dass obgleich bapin als pronomen im aschw. sehr geläufig ist, man fast ausschliesslich babe (nicht babin $\left.{ }^{1}\right)$ ) als conjunction in der verbindung bape - ok (sowol - als auch) benutzt. Dies ist leicht zu begreifen: um den begriff beides - und konnte man zwar beide - und aber nicht beide die - und anwenden.

2. Isl. nom. und acc. pl. neutr. $p a u, t v a u$, agutn. paun, aschw. pon, passin.

In Pauls Grundriss $I(\S 184,13)$ ist Noreen der meinung, dass isl. pau, aschw. po sich zu got. pō verhält wie skr. $t \bar{a} u \mathrm{zu}$ $t \bar{a}$ : 'd. h. wir haben hier ohne zweifel die alte form des nom. dual. $m$., welche in folge seines auslautenden $-u$ als nom. acc. pl. ntr. aufgefasst wurde und die ursprüngliche form verdrängte'. Die runische form $b a$ (nom., acc. pl. neutr.) ist nach ihm unerklärt. Nom., acc. neutr. tvau wird ib. $\$ 195,3$ in derselben weise wie bau gedeutet, wenn es heisst 'tuau ist wol der alte nom. masc. (skr. $d v \bar{a} u$; vgl. pau $=$ skr. $t \bar{a} u$..) der wegen des auslauts als nom. ntr. aufgefasst wurde'.

Ich finde diese erklärungsversuche nicht wahrscheinlich. Es scheint mir nämlich sehr kühn anzunehmen, dass die neutralen bau, tvau durch ein missverständnis einer masculinen form entstanden sein sollen, und wenn man auch annehmen wollte, das duale neutr. tvau sei durch ein missverständnis des dualen $d v \bar{a} u$ entstanden, so wäre es doch sehr auffallend, wenn das duale masculine tāu als eine plurale neutrale form aufgefasst werden konnte. Hierzu kommt noch, dass bei diesen erklärungen bau und tvau ganz von got. $p \bar{o}$ und twa getrennt werden, und dass das runiscbe pa.(nom., acc. pl. neutr.) unerklärt bleibt.

Folgende auffassung scheint mir diese schwierigkeiten $\mathrm{zu}$ beseitigen.

Man hat während einer fruheren periode im Norden nom. acc. neutr. twa, mit got. twa identisch, gehabt. $\mathrm{Zu}$ dieser zeit hatten nom. acc. pl. neutr. der starken adject. noch das auslautende $-u\left({ }^{*} g \bar{o} \delta u\right.$ etc.) erhalten, und nach ${ }^{*} g \bar{o} \delta u$ etc. und be-

1) Söderwalls aschw. wörterbuch enthält nur ein beispiel von bodenn als conjunction. 
sonders nach dem zahlwort friu (damals noch prīu ausgesprocben) nalm twa die form twau (ursprüglich $t w a-u$ gesprochen) an. Weil prīu, twa-u sich zu priú, twaú entwickelten, crhielten sie das auslautende $-u$, das aber in *gōðu etc. wegfiel ${ }^{1}$ ).

Im anschluss hieran erkläre ich $p a u(n), p \theta(n)$ folgendermassen. Bekanntlich ist $\bar{o}$ in unbetonter stellung in $\bar{a}, \breve{a}$ ubergegangen, so z. b. in der superlativ-endung -astr (got. armōsts : isl. armastr), und so auch in dem öfters proklitischen oder enklitischen acc. sg. fem. got. $p \bar{o}>$ isl. $p a \dot{~(F a l k, ~ A r k i v ~ N . ~ F . ~}$ II, 113). Der got. nom. acc. pl. neutr. $p \bar{o}$ bekam dieselbe entwickelung, und $p a$ ist runisch belegt. Von *gōðu etc. ubernahm $p \bar{a}$ die endung $-u: p \bar{a} u$, wonach $\bar{a} u$ in $\breve{a} u$ ubergieng und dieselbe behandlung wie der gewöhnliche diphthong au bekam (aschw. po). Vgl. dass westgerm. $\bar{a}+u$ im ags. durch verkürzung von $\bar{a}$ zu éa (néan aus *nă(h)un etc.) entwickelt worden ist, ebenso wie westgerm. $\breve{a}+u$ den ags. diphthong éa ergab (Sievers: Ags. gramm. $\left.{ }^{2} \S \S 112,111\right)$. Ich habe hier vorausgesetzt, dass der $a$-laut in $b a$ noch lang war, als es die form pau annahm; vielleicht war aber das lange $a$ in $p a$ bei der umbildung in bau schon in unbetonter stellung verkurzt worden, obgleich $p \bar{a}$ noch immer die betonte form war.

Die häufigste aschw. form ist aber nicht po sondern pon, agutn. paun, und Noreen fragt (ib. $\S 184,13$ ), ob pon, paun eine contamination vou pau und pen sei. Ich glaube dass diese frage verneinend beantwortet iverden muss. Agutn. paun verhält sich zu isl. pau wie aschw. passin (nom. acc. pl. neutr. und nom. sg. fem.) zu isl. aschw. pessi, poessi. Haben wir in bassin eine zusammenschmelzung von bossi + in (dem artikel)? Im isl. begegnet man oft z. b. pessi inn skakkborni sveinn; benna inn unga dreng; und auch z. b. sá inn fráni ormr; bat it mikla men; bann inn alsvinna iotun; born pau in blibu; bau in har jmóbgu ský etc. (belegstellen z. b. im Oxforder-wörterbuch s. 734 sp. 2 unter pessi B; s. 263 sp. 1 mom. II, 1). Aus passi-in konnte passin werden ebenso wie aus riki + in rikin etc. Die

1) Diese erklärung von tvau ist von anderer seite in einer (ungedruckten) abhandlung im philol. seminar der nniversität Lund gegeben worden. 
angeführten beispiele zeigen, dass auch nach sá, pau sehr oft (und zwar schon im ältesten isl.) der artikel inn, in folgt. Aus bau in entwickelte sich faun, und vielleicht am ehesten, schon als der hauptton noch auf a ruhte ( $b \dot{a} u)$; vgl. augu-in $>$ augun, tunga + in $>$ tungan; aber auch $p \dot{o}$-at $>$ bótt etc.

3. Isl. siau, siø. Noreen vergleicht (ib. § 204) isl. siau : aschw. sīu mit isl. tvau : aschw. $t \bar{u}$, und findet das spätisl. sio unerklärt. Da aber, wie soeben gezeigt, isl. tvau eine analogiebildung ist, mögen folgende bemerkungen uber siau, sio hier ihren platz finden. Es ist eine allbekannte tatsache, dass die zahlwörter oft sich gegenseitig beeinflussen. Ich erinnere $z$. b. nur an das dialektische aschw. ficerpia (statt ficerpa aus ficerpe) nach pripia (Kock, Arkiv N. F. II, 33 note). Man hat fruher *seun (aus *sebun) mit hauptton (fortis) auf der ersten, und starkem nebenton auf der zweiten silbe gehabt, was daraus zu erklären ist, dass das wort urspr. oxytoniert war (skr. saptá, gr. $\varepsilon \pi \tau \dot{\alpha})$, und dies hat das 'umspringen der quantität' (und des accents) im aschw. sī hervorgerufen (Sievers, Beitr. VI, 310 note). Durch anschluss an *ahtuu (got. ahtau) bekam *seun die nebenform *seau, welche aber die betonung von *seu $(n)$ beibehielt. Ebenso wie *seu(n) aschw. siū (mit hauptton auf $\bar{u}$ ) wurde, versetzte *seau den hauptton auf den diphthong au (siaú). Dies erklärt dass siau den diphthong, der in ahtau $>$ átta $\left.{ }^{1}\right)$ in $\bar{o}>a$ libergegangen ist, erhalten. Wenn diese erklärung das richtige getroffen hat, so ergibt sich hieraus, dass das umspringen der quantität und des accents in siau, sī̄ alter ist als die lautentwickelung $a u>\bar{o}$ in relativ unbetonten endsilben.

Im spätisl. (neuisl.) sio sehe ich die lautgesetzliche entwickelung von altisl. siau. Nachdem die lautverbindung au sich $z u \quad \ddot{j} j$ (in auga etc., als öjga etc. ausgesprochen) entwickelt hatte, ist $\ddot{o j}$ in der regel unverändert geblieben; man scheint aber nicht beobachtet $\mathrm{za}$ haben, dass $\ddot{o j}$ in der verbindung $j \theta j$ (durch eine art dissimilation) in $\ddot{o}$ ubergegangen ist. Dies ist

1) Die lïnge sowol des wurzelvocals als des t-lautes in aitta (aus ahtau) etc. kann wol nur so erklärt werden, dass zuerst der vocal vor $h t$ lautgesetzlich gedehnt wurde ( $\left.{ }^{*} \bar{a} h t a u\right)$, wonach später $h t$ in $t t$ assimiliert wurde (ătta). 
der fall nicht nur in siau $>$ sis sondern auch in iaur ' $\mathrm{ja}$ ', das (nach dem Oxforder-wörterbuch) zur zeit Gudmund Andreæ's jar ausgesprochen wurde, und die lautverbindung iöj durfte kaum in andern wörtern begegnen. Die spätisl. ordipalzahl sioundi hat $u$ von niundi, tiundi ubernommen. Wen $\mathbf{n}$ der $u$-laut in isl. siundi lang war, so ist es die normale entwickelung aus der cardinalzahl *setun ebenso wie aschw. siūnde.

4. Agutn. tyggia (gen. pl.). Der y-laut dieser genetivform ron tveir ist nach Noreen (ib. $\S 195,4$ ) auffallend. Folgende erklärung scheint mir befriedigend zu sein. Gen. pl. von prir lautet aschw. neben priggia auch pryggia: der $y$-laut ist aus nom. acc. pl. neutr. pry tibertragen worden. In ähnlicher weise kann tyggia aufgefasst werden. Zur zeit, als nom. acc. fem. $t v \bar{a} r$, acc. masc. $t v \bar{a}$, nom. acc. neutr. $t \bar{u}$ und gen. *tvaggia lautete, bildete man nach der analogie tvār, tvā : *tvaggia zu $t \bar{u}$ den neuen gen. *uggia, der durch den jüngeren $i$-umlaut spïter tyggia wurde. Da aber tveggia für alle genera gemeinsam war, wurde tyggia auch für masc. und fem. gebraucht, ebenso wie pryggia fur masc. gebraucht werden kann. Eine bestätigung hiervon gibt vielleicht das aschw. des festlandes, wo der gen. von tver tvaggia heisst, aber das compositum annattyggia geläufig ist: -tyggia ebenso wie annat ist neutrum.

5. álikr. Das aschw. aliker 'von derselben beschaffenheit', das auch, obgleich selten, im isl. (álikkr) begegnet, wird von Rydqvist, Sv. sprakets lagar II, 532 als pron. angefuhrt, und zum teil mit recht. Es ist aber nicht, wie or meint, mit der praep. $a$ zusammengesetzt, sondern mit der partikel, die isl. $e i$ 'immer' heisst, und welche als ein verstärkendes element mit adj. componiert wird, z. b. eilitill 'sehr klein'; vgl. auch eigrónn 'immergrün', Eirikr etc. ālīkr (eigentl. 'sebr gleich') hat den hauptton auf dem zweiten compositionsglied gehabt, und ai ist hier wie sonst in relativ unbetonter silbe (d. b. in silbe mit starkem nebenton) lautgesetzlich in $\bar{a}(\breve{a})$ ubergegangen (siehe uber diese entwickelung in óláfr : Ôleifr, afráb : aschw. afrezl, at 'nicht' : eitt etc. Kock: Svensk akcent II, 341 und jetzt auch Falk, Arkiv N. F. II, 114 note).

Ein paar andere pronominalformen mögen hier angefuhrt werden, wo die lautentwickelung $a i>a$ in unbetonter stellung eingetreten sein kann. 
Der vielbesprochene urnord. acc. pl. fem. paiaR (Istaby) kann im isl. pér, aschw. par fortleben. Aus paiaR wurde ${ }^{*} b \bar{a} a R>b \bar{a} R$, und aus $b \bar{a} R$ in betonter stellung durch $R$-umlaut isl. pér. Hiergegen kann nicht geltend gemacht werden, dass der dem ai (in baiaR) folgende vocal den $i$-laut gestlutzt haben muss. Der diphthong $a i(e i)$ ist nämlich aschw. nicht nur in beit $>b \bar{e} t$ etc. sondern auch (Noreen ib. $\S 141, \mathrm{~b}$ ) in isl. blaia $>$ aschw. blëa zu $\bar{e}$ geworden, und dies spricht dafur, dass auch während ciner älteren sprachperiode ein dem diphthong ai folgender vocal die normale entwickelung des diphthonges nicht verhinderte. Im isl. pére, aschw. par kann also sowol got. $p \bar{u} s$ als urnord. paiaR stecken.

Noreen (ib. 184, 11) fragt mit grossem zweifel, ob nom. pl. masc. isl. aschw. péer von fem. pér herrühre. Eine solche iibertragung einer fem. form ins masc. ist zwar möglich, aber doch wenig wahrsebeinlich. Es ist aber sebr wol möglich, dass nom. pl. masc. pai (got. pai) in unbetonter stellung * ${ }^{*} \bar{a}$ wurde, wonach dies durch ubertragung $-R$ bekam, ebenso wie aus der betonten form *bei peir wurde; als * $p \bar{a} R$ später auch nit hauptton ausgesprochen wurde, so wurde es in pér umgelautet. Der ubergang $p a i>{ }^{*} p \bar{a}$ ist der entwickelung got. bai $>$ ahd. the in unbetonter stellung (Braune: Ahd. gramm. $\S 43$ anm. 3) ganz analog, und der aschw. Uhergang $p \bar{e} r$ 'sie' $>h \breve{i}(r)$, neuschw. $d i$ bei verkillzung in unbetonter stellung bietet auch eine parallele.

III. Die genetiv-endung $-u(r)$ der starken fem.

Im adän. des zwölften jahrhunderts, also aus einer vorliterarischen zeit, ist diese endung in mehreren personennamen belegt. 0 . Nielsen: Olddanske personnavne (1883) s. XI teilt aus dem 'Necrologium lundense', einer schonischen urkunde, beispiele mit; diese adän. endung ist aber, wie Noreen in - Pauls Grundriss § 172, 2 bemerkt, ebenso wie -ur (-or) im anorw. laugurdagr, aschw. loghordagher unerklärt ${ }^{1}$ ).

1) Aus gutem grunde billigt er also die ansicht K. Piehls (Tidskrift f. Filologi, N. R., V, 281 note) von aschw. leghordagher nicht. Piehl meint, leghordagher sei eine contamination von leghardagher und leghodagher, und in loghodagher soll $o$ (ebenso wie in kopoiorp etc.) analogisch eingefuhrt sein. Obgleich diese auffassung vom aschw. leghor - 
Die von Nielsen a. a. o. ver\%eichneten wörter mit der gen.endung - $-u$ sind Thorunn (isl. I'órunnar), Gunnuru (isl. Gunnvarar), Olouo (isl. Ólofar), Islogu (isl. *Íslaugar), Seburgu (vgl. isl. Sćbiargar), Sifrithu, Sestrithu (vgl. Nielsen s. 81), und hierzu kommt noch Thrugunnu (auch in der zusammensetzung Thrugunnuson; isl. Porgunnar, rgl. Nielsen s. 94). Eine allgemeine ïbertragung aller dieser namen aus der $a$-in die $n$-declination dürfte nicht angenommen werden können. Hiergegen sprechen nämlich erstens das hohe alter der gen. auf $-u$, zweitens dass keine den gen. Thorunnu etc. entsprechenden nom. auf $-a$ von Nielsen verzeichnet sind; hierzu kommt noch, dass bei dieser auffassung von Thorunnu etc. das anorw. laugurdagr unerklärt bleiben würde. Alle diese namen ausser Sifrithu, Sestrithu haben aber in der der endung $-u$ vorangehenden silbe einen $u$-(o-)laut, und dies ist auch im anorw. laugurdagr der fall. Ich setze dies mit der auffallenden gen.-endung in causalverbindung und nehme an, dass (wenigstens in gewissen gegenden) der urnord. endrocal $\bar{o}$ als $o, u$ blieb, wenn die vorhergehende silbe einen $u$-oder $o$-laut enthielt. So gieng z. b. der gen. ${ }^{*} \bar{\imath} d \bar{o} R$ in tipar uber, aber gen. ${ }^{*} \bar{p} \bar{r}$ unno $R$ wurde als Thorunnu( $r$ ) erhalten. (Der verlust von $-r(-R)$ im adän. des zwölften jahrhunderts stimmt mit dem gleiclszeitigen verlust von $-r(-R)$ im gen. $-a(r)$ : Thormotha aus Thormothar etc. ubercin, vgl. Nielsen s. X.)

Ich sehe in der erhaltung von $o(u)$ eine art vocalbarmonie, und êliêsıs wie die gewöhinliche vocalharmonie $u: o, i: e$ in anorw. und altschon. urkunden begegnet (siebe Kock, Arkir N. F. I, $79 \mathrm{ff}$. und die daselbst angeführte literatur), so ist es vorzugsweise in Norwegen und Schonen, wo die erhaltung von $o(u)$ nach $u(o), a u$ angetroffen worden ist. Es verdient auch bemerkt $z u$ werden, dass ebensowie der den letzteren componenten des diphthonges au ausmachende $u$-laut den endvocal im anorw. laugurdagr conserviert hat, nicht nur $u, \dot{u}$ sondern auch der diphthong au nach dem anorw. vocalharmoniegesetz den endvocal $u$ fordert, z. b. lausum, ruddu, husum.

dagher denkbar wäre, kann sie nicht acceptiert werden, da sie anorw. laugurdagr nicht erklärt: kein anorw. laugudagr ist meines wissens belegt.

Beitrăge zur geschichto der deutschen sprache. XV. 
Man versteht leicht, warum der labiale endvocal gerade in fem. personennamen und im worte laugurdagr bleibt. Im allgemeinen haben wörter mit den wurzelvocalen $u, o$ die endung -ar durch anschluss an andere wörter angenommen; so z. b. gen. laugar nach gen. reimar etc. In der zusammensetzung laugurdagr entzog sich aber der gen. laugur- diesem einfluss, und bekanntlich werden nomina propria leicht als isolierte gruppen aufgefasst und erhalten (resp. bekommen) deshalb eine von der gewöhnlichen abweichende flexion.

Dies kann z. b. der fall mit den unumgelauteten personennamen auf -arr (Gunnarr etc.) im gegensatz zu herr etc. (Noreen, Bezzenbergers Beitr. XI, 196, Streitberg, Beiträge XIV, 179) gewesen sein, und dies ist mit den aschw. fraueunamen auf -borgh der fall. Obgleich das einfache $\operatorname{borg}(h)$ im isl. und aschw. ein $i$-śtamm (pl. isl. borgir, aschw. borghir [und borghar]) war, so haben im ascliw. Ingeborgh, Ramborgh etc. nach den zablreichen frauennamen, die als $j a$-stämme flectieren, in dat. und acc. die endung - $e$ angenommen ${ }^{1}$ ).

Die adän. frauennamen mit $u(0)$ in zweiter silbe (Thorun etc.) können um so leichter als eine isolierte gruppe gefiblt worden sein und eine ältere flexion erhalten haben, weil sic sehr zahlreich waren. Folgende mögen, ausser den schon erwähnten, aus der schrift Nielsens verzeichnet werden: Allogh (fruber *Allaug; und Allog), Arylogh, Aslogh, Finlogh, Gerlogh, Gunlogh, Ketillogh (und Koethellug), Swanlogh, Thorlegh; Almgun, Asgun, Hildegun; Gerborgh, Helinburgh, Hildeburgh, Ingiburgh, Odburgh, Regnburgh, Wiburgh; Guthrun; Ermentruth; Biarund.

Die zwei gen. Sifrithu, Sestrithu mit $i$ in penultima brauchen besonders besprochen zu werden. Man kann sie in verschiedener weise auffassen. $\mathrm{Da}$, wie wir soeben gesehen haben, die frauennamen mit $u(o)$, au in der letzten wurzel-

1) Einige belegstellen aus dem anfang des 15. jahrhunderts mögen angeführt werden. Dat. und acc. Ingeburghe (Svenskt diplomatarium N. S. III nr. 2400, Vadstena). Dat. Ingeborghe (ib. II nr. 1025 und nr. 1479). Dat. Gunborghe (ib. nr. 1379). Dat. Ramborghe Joansdottor (ib. 1143). Vgl. die häufigen dative von ja-stämmen Ragnilde (ib. nr.1143), Ingegorpe, Gerprupe, Ingripe etc. und die zahlreichen frauennamen, die im isl. diese flexion haben: worter auf $-r$ (Gerpr etc.), -unn (Ipunn etc.), -dis (Fordis etc.). 
ilbe im adän. sehr zablreich waren, so können Sifrith, Sestrith ich an ihre flexion angeschlossen haben, ebenso wie z. b. die :schw. Ingeborgh, Gunborgh etc. die flexion der zahlreichen rauennamen, die ja-stämme sind, angenommen. Aber auch ine andere erklärung ist möglich. Man besitzt im adän. eine nenge frauennamen auf - $a$ im nom., die unter derselben form $\mathrm{m}$ ahd. begegnen und wenigstens zum teil aus dieser sprache intlehut sind, z. b. ahd. adän. Apa, Awa, Ida, Imma, Inga, reta, Una, ahd. Wiba, adän. Wiwa. Diese namen waren im :dän. ohne zweifel $n$-stämme und giengen im gen. auf $-u$ aus. )a nun, wie Nielsen s. 82 bemerkt, ahd. Sigifrida dem adän. jigfrith, das masculine ahd. Sisifrid dem adän. fem. Sestrith intspricht, und da das ahd. auch den frauennamen Frida, der im :dän. ortsnamen Frithuthorp (Nielsen s. 27) begegnen kann, ils simplex besitzt, so ist es wol auch möglich, dass, ebenso vie man ahd. adän. Apa, Awa etc. hatte, das adän. gerade von sifrith, Sestrith, die im deutschen nahe verwante batten, die rebenformen Sigfritha, Sestritha, die als $n$-stämme flectierten, iesass. Vgl. besonders dass (nach Nielsen s. 3) Alvgun, welhes dem ahd. Albagunda entspricht, etwas später (aus dem 3 ? jahrhundert) in der form Algunno belegt ist ${ }^{1}$ ).

Durch die obige erklärung der gen.-endung $-u(r)$ wird rielleicht auch uber ein paar andere fragen licht verbreitet. is ist auffallend, dass in wrta (1. pers. sg. praet.) in der inichrift der Etelhemspange ${ }^{2}$ ) der endrocal $\bar{o}$ des älteren $n o-$

1) An diesem orte mag eine andere auffallende endung der adän. versoneunamen erwähnt werden. Mannsnamen auf vin haben in miinzegenden des elften jahrhunderts oft die form -vini, -vine; z. b. Sevvini, rivvine, Godwine, Alfwine. Dies sind jedoch keine uralten adän. formen. nit erhaltenem endrocal, sondern sie rühren von englischen münzneistern her, die die ags. namensform auf -wini, -wine benutzt haben.

$\left.{ }^{2}\right)$ Ueber die deutung dieser inschrift siehe Noreen in Bezzenbergers 3eiträgen XI, 201 und Kock, Undersökningar i svensk språkhistoria $108 \mathrm{ff}$. ch lese die inschrift ek Erla w(o)rla 'ich Erla (aschw. Jarle) machte die spange)'. Die urnord. nom.-endung - $a$ der masc. $n$-stämme ( ${ }^{*} t$ trma, Lirla, Wivila etc.) ist nach meiner ansicht lautgesetzlich in $-i$, $-e$ uber; egangen (isl. timi, aschw. Jorle etc.). Im unbetonten auslaut (nicht ıber im inlaut) dürfte die entwickelung $a>i$ lautgesetzlich eingetreten sein. Vgl. dass im aschw. unbetontes $-i$ auslautend $-e$ wird, inlautend tber bleibt (timi $>$ time, aber timin etc.). 
rahto (auf dem Tune-stein) schon a geworden ist, obgleich man auf dem viel jüngeren Björketorp-stein noch runo (und auch auf dem Stentofta-stein runono) begegnet. In worahto $>$ vrta ist $\bar{o}$ wegen des svarabhaktivocals in $a$ ubergegangen, in runo (runono) aber durcb den $u$-laut der vorhergehenden silbe erbalten. Jetzt versteht man auch, wie trotz runo auf dem Björketorp-stein der ungefähr gleichzeitige Istaby-stein runaR haben kann: die endung -ar dieser form ist von wörtern ohne $u(o)$ in der wurzelsilbe tibertragen worden.

Die nom.-endung -ur in den aschw. mopur, brobur, doctur (=dottur), fabur, adän. mobur, bropur, fapur ist von Noreen (Språkvetenskapliga sällskapets i Upsala förhandlingar 18821885 s. 124, Pauls Grundriss § 36 a) mit einigem zweifel (die nom. mopur etc. kōnnen nämlich aus den casibus obliquis uber-

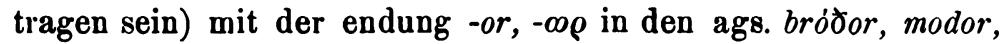
dohtor, sweostor und im gr. (dor.) $\varphi \rho \alpha ́ \tau \omega \rho$ identificiert worden. Der $u$-laut in den ostnord. mopur etc. macht aber schwierigkeit, da man nach den gewöhnlichen lautgesetzen $a$ (nicht $u$ ) erwartet, und Noreen ist deshalb geneigt anzunehmen, dass $o(u)$ in den altnord. sprachen lautgesetzlich auch vor urspringlichem $r$ bleibt. $\mathrm{Da}$ aber dies gesetz ausschliesslich oder fast ausschliesslich auf den angefluhrten verwantschaftswörtern fussen wurde, so ist es einfacher anzunehmen, dass, wenn alte nom. in mopur etc. vorliegen, der ursprungliche o-laut als $u(o)$ in mopur, bropur, doctur (dottur) wegen des vorbergehenden o-lautes erbalten ist, und dass fajur an die eben genannten verwantschaftswörter angeschlossen wurde, so dass $u$ auch hier blieb. Auch sonst hat nämlich fapir dialektisch im aschw. den endvocal angenommen, der lautgesetzlich nur den ubrigen verwantschaftswörtern zukam: man begegnet in ur. kunden mit vocalbalanz fur $u: o$ als gen. dat. acc. fapor (statt fapur) durch anschluss an mopor, bropor, dottor, systor (Kock: Studier öfver fsv. ljudlära I, 178).

IV. 2. pers. pl. auf -r (anorw. -ir, -ur; ostnord. -er in der 2. pl. imper.).

Aeltere sprachforscher wie z. b. Gislason (Um frumparta isl. tungu s. $214 \mathrm{ff}$.) und Lyngby (Annaler f. nord. oldkyndighed 
1854 , s. 223; Antiqvarisk Tidsskrift $1858-60$ s. 270 f.) waren der ansicht, dass $-r$ in dieser endung sich aus älterem - $\delta$ entwickelt, so dass pracs. bindid, praet. bunduð die anorw. bindir, bundur, ebenso wie pl. imperat. bindið das adän. aschw. binder, gegcben hätten ${ }^{1}$ ). Als aber diese meinung herrschte, machte man kleinere ansprïche an die consequenz der lautgesetze als heutzutage, so dass man keinen anstoss daran nahm, dass obgleich bindið bindir ergeben hatte, der $\delta$-laut in blí etc. immer hlicb. Es ist wol dieser umstand der Läffler veranlasst hat in 'Tidskrift f. Filologi, N. R., V, 78 eine andere erklärung von anorw. $-i r$ vorzuschlagen. Nach ihm soll $-r$ in den verbalcndungen -ir, -ur von dem pron. ér, pér stammen (ér, pér bindið $>\dot{e} r$, pér bindir). Diese erklärung kann jedoch als keine befriedigende angesehen werden, denn einerseits ist die von Läffler vorausgesetyte angleichungr, wenn auch möglich, doch ziemlich auffallend, andrerseits wird die ostnord. imperativendung -er dadurch nicht erklärt. Denn der ostnord. imperativ wird in der regel vou keinem pron. pers. begleitet. - Mit recht hat also Noreen (in Pauls Grundriss §229) die ansicht Läfflers nicht acceptiert: nach ihm ist die endung -ir unerklärt.

Ich werde aber hier nachzuweisen versuchen, dass $-r$ in der 2. pers. pl. die direkte entwickelung aus älterem $\partial$ ist, welche erklärung naturlich am nächsten liegt, wenn die lautgesetze sich damit vereinigen lassen.

In verschiedenen nord, lịalekten ist $\delta$ in allen stellungen lautgesetzlich in $r$ übergegangen. Dies ist z. b. der fall in gewissen dialekten von Dalarna (Lundell, Svenska landsmålen 1, 153), im Kalixdialekt (Widmark: Vesterbottens landskapsmål s. 13), in jütländischen dialekten (dän. mað 'speise' > mar etc., Thorsen: Nörrejysk lydlære $67 \mathrm{f}$.). Die ostnord. literatursprachen zeigen den übergang $\dagger>r$ vor $k$ in z. b. aschw. madhker $>$ marker, budhker $>$ neuschw. burk, adän. odhken $>$ orken. Auch in isl. und besonders in anorw. handschriften trifft man spuren ciner entwickelung von auslautendem $\partial$ in $r$ (siehe Gislason, Annaler for nord. oldkyndighed 1858 , s. 136 note 6 ; Wiséns

1) Die im späten aschw. und im (älteren) neuschw. begegnende imperativ-endung -er ist vielleicht eine dïnische entlehnung. 
Homiliu-bók s. XI, Noreen in Pauls Grundriss $§ 112$, c). Unter diesen umständen wäre es sonderbar, wenn das auslautende $r$ in anorw. bindir, adän. binder (dem isl. bindið gegenuber) in keiner beziehung zum anorw. dialektischen lautlubergang $\partial>r$ oder zur dän. dialektischen entwickelung $\delta>r$ stände. Ich nehme deshalb an, dass während $\delta$ nur dialektisch in allen stellungen $r$ wurde, im anorw. und in den ostnord. sprachen liberhaupt folgendes gesetz sich geltend gemacht hat: silbenauslautend geht $\partial$ in relativ unbetonter silbe in $r$ uber. Bei dieser abfassung der regel versteht man, warum in den meisten gegenden blidh, kalladhe etc. den $\gamma$-laut erhalten, obgleich er in bindir $r$ geworden ist.

Wenn es nachgewiesen werden könnte, dass $\partial$ dieselbe entwickelung in zusammensetzungsgliedern, die den bauptton entbehren, bekommen hat, so wüde dies eine willkımmene bestätigung des lautgesetzes bieten. Dies ist auch in der tat der fall gewesen. Aus aschw. *spadhlimaper entwickelte sich teils sperrlemmad (im älteren neuschw., d. h. spärlémmad), teils spädlemmad, aber nur spadher $>$ späd. Aschw. hādhvéta 'schmähen' aus hâdh 'bohn' ist in mehreren gegenden, wo, so viel man weiss, $\delta$ sonst nicht in $r$ ubergeht, harveta geworden. Die betonung hädhvéta wird durch den $a$-laut in der ersten silbe von harveta bestätigt: in hâdhveta ist der lange $a$-laut vor dem lubergang $\bar{a}>\grave{\bar{a}}$ verküzt worden, weil der hauptton auf dem zweiten compositionsglied ruhte (vgl. dän. amindelse aber $g a ̊, p a ̊$ etc.). So muss auch isl. vapmál, aschw. vapmal (mit $v \bar{a} b$ 'a piece of stuff' componiert) $>$ varmal (in norw. dialekten) $>$ aschw. valmar erklärt werden ${ }^{1}$ ). Vadhmal mit hauptton auf ultima wurde varmal und durch metathese von $r$ und $l$ valmar; vgl. z. b. aschw. vanmal > valman, genom : gymon, gemen (Rydqvist IV, 453 f.), hoeleda, hoclede ('beld') > hoedela, haedele (Rimkr. II; vgl. ib. s. 384), den ortsnamen Valora: Ia rola; im adän. min 'mein' $>$ nim (so mehrmals in der sprichwörtersammlung Peder Laales von 1515). Zweifelhaft ist es ob im vereinzelten annorghom (im Vestgötalagen I) statt an-

1) Ich kann mich der meinung Noreens (Svv. landsmålen I, 359), der varmal, valmar aus *vaparmal erklärt, nicht anschliessen, denn kfin *va parmal ist belegt. 
nofghom und im neuschw. merafton (aschw. mipapton) ein tibergang $\partial>r$ vorliegt (vgl. Rydqvist IV, $328 \mathrm{f}$.); denn dies kann auch aus mipr apton entstanden sein. In zwei runeninschriften (Liljegren nr. 1049 und 1131) begegnet die praeposition map unter der form $m i R^{\prime}|m \propto R|$, das doch wol aus mäðR entstanden ist. $\left.{ }^{1}\right)$

Dem hier angenommenen lautgesetz widerspricht natülich der umstand nicht, dass man keinen (wenigstens durchgeführten) übergang $\partial>r$ in nom. sg. fem. kalluð etc. oder in acc. sg. skilnað etc. begegnet. Der $\partial$-laut ist in solchen formen durch anschluss an die ibrigen casus mit lautgesetzlichem $\delta$ (kallaðr, kallaðir etc.; skilnaðar, skilnaði etc.) erhalten worden.

V. Zur umlauts-und betonungsfrage.

An die von mir (Arkiv IV, $141 \mathrm{ff}$. = Beitr. XIV, $53 \mathrm{ff}$.) dargestellte theorie für den $i$-umlaut, die von Noreen in Pauls Grundriss $\S 49$ ganz acceptiert worden ist, hat sich Heinzel (Anzeiger für deutsches Altertlium XIV, 219 note) wesentlich angeschlossen, wenngleich er einigen zweifel betreffs meiner mit der umlautstheorie verknupften ansicht von der vorliterarischen anord. betonung äussert. Ich werde mir einige bemerkungen erlauben um darzulegen zu versuchen, dass die theorie von der gemeinnord. betonung so innerlich mit der vom $i$-umlaut verbunden ist, dass man, wenn jene gebilligt wird, folgerecht auch diese acceptieren muss, und dass meine auffassung, den a. a. 0, dargestellten bedenklichkeiten des hervorragenden germanisten gegenliber, aufrecht erbalten werden kann. Ich glaube auch das wolwollen des redacteurs dieser

1) Ueber die wechselnden formen des wortes vapmal kann noch folgendes bemerkt werden. Bei der betonung vápmal wurde $\partial$ (später d) erhalten: neuschw. valmal; dialektisch aber gieng $\delta$ durch partielle assimilation in $n$ über: aschw. vanmal ( $\mathrm{vgl}$. in Fryksdaldialekt im vorigen jahrhundert nach Noreen, Sv. landm. I, 190 Gunmun aus Gudhmund). In einigen gegenden war die assimilation von $\partial m$ vollständig: neuschw. vammal (vgl. gud/hmor > gummor). Vanmal wurde volksetymologisch an vander 'stock' angeschlossen: aschw. vandmal (vgl. isl. alin 'a unit of value, viz. an ell of woollen stuff [vapmál]'). Aschw. valmal ist eine contamination von vanmal und valman; aschw. valmal (z. b. Svenskt diplomatarium, N. S. II s. 699 ; auch im älteren neuschw. geläufig) ist eine niederdeutsche form (mnd. watmal). 
zeitschrift kaum misszubrauchen, wenn ich jetzt auf diese frage zurlickkomme, da sie fur die gemeingerm. betonungslehre von yrosser bedeutung sein durfte.

$H$. bezweifelt dass die aschw. und anorw. vocalbalanz, die eine hauptstutze meiner theorie von der vorliterarischen betonung ausmacht, notwendig von der betonung hervorgerufen sei.

Diese bemerkung H.'s dürfte wol einem missverständnis zuzuschreiben sein. Die frage steht ja folgendermassen. Die gemeinnord. endvocale $a, i, u$ sind im aschw. (und zum teil auch im anorw.) nach der vocalbalanz so behandelt worden, dass sie in zweisilbigen wörtern mit kurzer wurzelsilbe bleiben, in zweisilbigen wörtern mit langer wurzelsilbe aber in resp. $a, e, o$ geschwächt worden sind; flŭgha, sp̌ni, flüghu bleiben unverändert; $d \bar{u} v a, t \bar{\imath} m i, d \bar{u} v u$ aber werden $d \bar{u} v a, t \bar{\imath} m e$, $d \bar{u} v o$. Da nun sehr altertumliche neunord. dialekte in Schweden (der dialekt von Elfdalen in Dalarna) und in Norwegen die wörter mit kurzer und diejenigen mit langer wurzelsilbe noch verschieden betonen, und zwar so, dass jene auf der zweiten silbe einen starken nebenton tragen, den aber diese entbehren, und da weiter gerade derselbe schwedische dialckt (in Elfdalen) die vocalbalanz $i: e, a: a^{1}$ ) noch erhalten, so kann es keinem zweifel unterliegen, dass ja die vocalbalanz von dieser betonung abhängt: der starke nebenton in flügha, spǐni, flŭghu hat die alten endvocale $a, i, u$ conserviert, welche in der ganz tonlosen ultima von dūva, tīme, dūvo in resp. ae, $e, o$ geschwächt worden sind. Und in der tat scheint $H$. selbst denselben gedanken, nur mit anderen worten, auszusprechen, wenn er äussert: 'da im letzteren falle (in ľ̃va) nur das ältere $a$ bleibt, nicht etwa ein laut eintritt bei dem eine grössere energie der aussprache wahrscheinlich wäre, so kann man sich die sache so vorstellen, dass in bīta wegen der längeren dauer der ersten silbe die energie der aussprache am ende des wortes schon so abgenommen hatte, dass man nicht mehr $a$ sondern $a$ producierte'. Dass die kleinere energie, womit die ultima von bìta ausgesprochen wurde, den ibergang $b i ̄ t a>$

1) Die vocalbalanz $a: a e$ wird in diesem dialekt von $a: a$ repräsentiert. 
bītce (trotz lŭva, nicht lĭvce) möglich machte, ist, so viel ich sebe, nur ein anderer ausdruck dafür, dass der nebenton in Iiva den $a$-laut schützte, der aber in der ganz tonlosen ultima von bìta $>b \bar{t} t c e$ in $a$ geschwächt wurde.

Diese betonung war gemeinnordisch (vol. Kock, Beitr. XIV; 61), und da nach den untersuchungen von Sievers genau dicselbe betonung auch für das westgerm. galt, so habe ich (Beitr. $\mathrm{XIV}, 66 \mathrm{ff}$.) angenommen, dass der wegfall von $i$ in $* k v \bar{a} n i R>$ kvcén $(R)$ etc. und seine vorläufige erhaltung in *stadiR etc. von dieser westgerm.-gemeinnord. (d. b. gemeingerm.) betonung abhing. Dies will $\mathrm{H}$. nicht gelten lassen. Obgleich auch nach seiner meinung $i$ frïher nach langer als nach kurzer silbe wegfiel, und der ältere $i$-umlaut mit seinem wegfall verbunden war ${ }^{1}$ ), so soll der verlust des endvocales nicht von der betonung, sondern, wenn ich den verehrten verfasser richtig verstanden habc, von einer tendenz der sprache $* k v \bar{a}$ niR mit *stadiR quantitativ gleichwertig zu machen, hervorgerufen $\operatorname{sein}^{2}$ ), und dieselbe erklärung scheint er auf die westgerm. gast, fuoz im vergleich mit wini, sunu erstrecken zu wollen.

Dieses durfte aber nicht richtig sein. Zwei kurze silben können zwar metrisch einer langen gleichwertig sein; ich bin aber fest iberzcugt, dass ich, ohne mich zu irren, behaupten kaun, dass dem gewöhnlichen sprachgefuhl (in der alltäglichen prosaischen sprache, die hier naturlich die einzig massgebende war) zwei wörter wie die zweisilbigen *kvāniR und

1) Mit diesem $i$-umlaut in *kvāni $>k v \overline{e_{n}}(R)$ war auch eine zurïckwerfung des accentes der ultima in *kvänin verbunden, so dass kvēen $(R)$ einen zusımmengesetzten musikalischen und zweigipfligen exspiratorischen accent bekam, ebenso wie dieselbe betonung in mehreren älteren und jüngeren indogerm. sprachen (aind., griech., neuschw. und neudeutschen dialekten etc.) durch den verlust einer silbe entsteht (vgl. Beitr. XIV, 72). Auch der indugerm. (im griech. und litauischen erhaltene, vgl. Fr. Hanssen, KZ. XXVII, 612 ff.; Brugmann, Grundriss I § 671) circumflex dürfte einen ähnlichen ursprung haben.

2) H. äussert: 'Wenn *stapiz, *kvāniz, - *talida, *dōmida, */ukile, *angile einander gegenüberstanden, konnte in der ersten [i-umlauts-| periode auch das bedürnis nach gleichem gewicht der verwanten formen zum ausfall des $i$ in kvāniz, dōmida, angile und damit zum um-

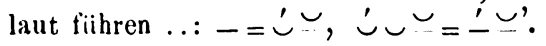


*stadir ähnlicher vorkamen als das einsilbige *kvēenR und das zweisilbige *stadiR. Uebrigens muss man sich noch erinnern, dass die wörter mit langer wurzelsilbe vielmal zahlreicher als diejenigen mit kurzer wurzelsilbe waren, und wenn

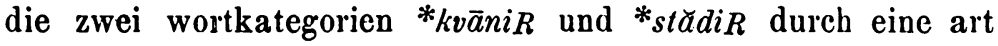
ubbertragung quantitativ gleichwertig gemacht werden sollten, würde man deshalb unbedingt erwarten, dass *stadiR die quantitätsverhältnisse von *kvāniR angenommen hätte, und nicht umgekehrt.

H. meint endlich, dass die $u$-synkopierung nicht parallel mit der des $i$ verlaufe, da das isl. logr : vollr aber stabr : kvén hat, und dass vallgangr abor logvellir beweisen soll, dass in der ersten umlautsperiode *vallugangr und logvellir neben einander bestanden haben, dass also $u$ nach kurzer, nicht aber nach langer silbe tendenz zum abfall und damit umlautwirkung gehabt hätte.

Wenn man aber etwas näher zusieht, lassen sich die tatsächlich belegten formen leicht erklären.

Es wird allgemein anerkannt, dass der endvocal $u$ etıvas später als $i$ wegfiel, und also muss der ältere $i$-umlaut fruber als der ältere $u$-umlaut eingetreten sein. Da weiter im anorw. der jungere $u$-umlaut noch nicht gewirkt hat, der jungere $i$-umlaut aber gemeinnordisch ist, so ist auch der jungere $i$-umlaut älter als der jungere $u$-umlaut. Da also der $i$-umlaut keineswegs gleichen schritt mit dem $u$-umlaut in der meinung hielt, dass ibre erscheinungen gleichzeitig durchgeführt wurden, so kann man gar nicht erwarten, dass z. b. stapr $i$-umgelautet sein müsse, weil logr $u$-umgelautet ist: der

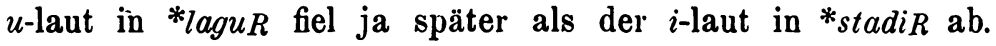
Was man höchstens erwarten kann, ist dass in den verschiedenen stellungen der abfall des endvocales $u$ in derselben reihenfolge eintrat, wie der abfall des endvocales $i$ in den entsprechenden stellungen schon etwas fruher eingetreten war, und so viel ich sehe, widersprechen die tatsachen dieser erwartung nicht. Das verhältnis durfte am besten durch folgende tabelle veranschaulicht werden, wo die formen nach dem chronologischen verlust des endrocales $i$ und nachher nach dem chronologischen verlust des endvocales $u$ verzeichnet sind. 
Verlust von $i$.

Anfang der älteren $i$-umlautsperiode.

*kvāni $i_{R}=k v a ́ n(k),{ }^{*}$ dōmido $>$ dómda.

Schluss der älteren $i$-umlautsperiode.

${ }^{*}$ stadi $_{R}>$ stapr, ${ }^{*}$ talido $>$ talpa.

*kvānifang $>$ kvánfang.

Anfang der jüngeren $i$-umlautsperiode.

suni $_{R}>$ synir.

*hariskip $>$ •heriskip; später herskip.

Verlust von $u$.

Anfang der älteren $u$-umlautsperiode.

${ }^{*}$ vallu $_{R}>$ vollr, "axula $>$ oxlar.

${ }^{*}$ lagu $u_{R}>\log r,{ }^{*}$ sadula $>$ soplar.

Schluss der älteren $u$-umlautsperiode.

*vallugangr $>$ vallgangr.

Anfang der jüngeren $u$-umlautsperiode.

*lagum $>$ logum.

*laguvelli ${ }_{R}>$ *loguvellir; später logvellir.

Hieraus ergibt sich, dass sowol $i$ als $u$ (obgleich zu verschiedenen zeiten) zuerst in simplicibus mit langer wurzelsilbe (kvén, vollr) wegfiel, nachher in simplicibus mit kurzer wurzelsilbe (stapr, logr), dann im ersten compositionsglied mit langer wurzelsilbe (kvánfang, vallgangr), und endlich im ersten compositionsglied mit kurzer wurzelsilbe (herskip, logvellir). Dass der abfall von $i$ in herfang verbältnissmässig sehr jung ist, und dass hier also der jlingere $i$-umlaut vorliegt, ergibt sich daraus, dass der unsynkopierte gen. Harinulfs noch in der spätei Räfsalinschrift begegnet, und dies wird vom isl. Heriolfr bestätigt, wo der verlust von $w$ vor $o$ eine notwendige voraussetzung für die bewahrung des $i$ ist (vgl. Noreen in Pauls Grundriss $§ 49,2$ ).

Wie schon Beitr. XIV, 67 note bemerkt worden ist, scheint $i$ in *kvānifang später als in *kvāniR abgefallen zu sein; der vergleich mit den $u$-stämmen spricht aber dafür, dass der abfall von $i$ in *kvānifang auch etwas jünger als der in $*_{\text {stădiR }}$ ist, und da bekanntlich auch in den anderen germ. sprachen die stammvocale länger im ersten compositionsglied als sonst erhalten werden, so spricht, so viel ich sehe, nichts dagegen. Asmund neben sunu auf dem Sölvesborg-stein kann auch leicht erklärt werden. Obgleich óss ursprünglich ein $u$-stamm war (Asugisalas auf der lanzenspitze von Kragehul), so wird schon 
früh im isl. áss (óss) nicht nur als $u$ - sondern auch als $a$-stamm flectiert (Wimmer, Fornnordisk formlära $\$ 51 \mathrm{~b}$ anm. 1), und Asmund braucht deshalb nicht unmittelbar aus *Asumund, kann aber aus *Asamund hervorgegangen sein. Besonders im ersten compositionsglied kann $a$ statt $u$ leicht durch anschluss an die sehr zahlreichen wörter mit $a$-stämmen als ersten compositionsgliedern übertragen sein; vgl. im gr. $i \chi \vartheta v-o-\varepsilon \iota \delta \eta^{\prime} s, \pi \alpha \tau \varrho-o-$ $x \alpha \sigma i \gamma \nu \eta \tau o s$ etc. mit libertragung von $o$ aus den 0 -stämmen.

Obgleich die obige tabelle den normalen chronologischen verlauf richtig veranschaulichen dürte, so wäre es wol doch nicht undenkbar, dass der verlauf in verschiedenen gegenden in einzelnen etwas verschieden gewesen wäre, besonders da mehrere von den vocaleinbussen jedenfalls ungefähr gleichzeitig eingetreten sein müssen, und es wäre wol auch möglich, dass der compositionsvocal $\mathrm{zu}$ verschiedenen zeiten weggefallen, je nachdem der hauptton auf dem ersten (*kvä́nifang) oder auf dem zweiten compositionsglied (*kvānifáng) ruhte. Hier ist natülich nur von der normalen entwickelung in den gegenden, wo die literatursprachen später entstanden, die rede gewesen.

Als eine gute stlitze meiner $i$-umlautstheorie mag noch folgendes hinzugefugt werden. Bekanntlich sind viele ursprüngliche $\bar{\imath} n i$-stämme, trotz der langen stammsilbe, nicht umgelautet, z. b. aupn, förn, lausn (got. lauseins), nautn, niósn (got. niuhseins), stiorn, pausn, aber heyrn (got. hauseins; vgl. z. b. Falk, Arkiv IV, 355). Mit der ălteren auffassung des $i$-umlauts können diese unumgelauteten $\bar{i} n i$-stämme nicht befriedigend erklärt werden. $\mathrm{Da}$ in allen casibus ein $i$-laut nach der wurzelsilbe folgte (nom. *fōrin, gen. *fōrinaR, pl. *fōriniR etc.), so sollte ja der $i$-umlaut ub erall eintreten, nicht nur in * fórinaR $>$ *fórnar (vgl. *dōmido $>$ dómda) sondern auch in * fórin $>$ *forin etc., und der tatsächlich im isl. begegnende unumgelautete vocal (förn) sollte nirgends bleiben. Wenu man aber zwei umlautsperioden annimmt, können die formen sehr leicht erklärt werden. Wie Bahder, Die verbalabstracta s. 84 und Bugge, Arkiv II, 213 dargetan haben, sind die einsilbigen nominativ-formen skirn (got. skeireins) etc. von den contrahierten casibus eingedrungen (früher nom. *skīrin, gen. skīr$n a R$ etc.). Während der älteren $i$-umlautsperiode bekam man 
nonı. acc. *fōrin, gen. *förnaR, pl. *förniR etc. Vor der durchführung des jüngeren $i$-umlauts drang der unumgelautete rocal in die lautgesetzlich umgelauteten casus ein (fōrnaR, förniR etc.), und nach gen. förnaR, pl. förniR etc. wurden nom. acc. sg. fōrn neugebildet, ebenso wie skírn nach skīrnaR etc. Heyrn aber ist unmittelbar nach dem lautgesetzlich umgelauteten gen. heyrnaR etc. gebildet worden. ${ }^{1}$ )

1) Correcturnote. In der jüngst erschienenen und mir jetzt zugänglichen abhandlung Fornnorska homiliebokens ljudlära I von Elis Wadstein wird (s. 5S) anorw. aschw. poer 'sie' (nom. pl. masc.) aus peir in unbetonter stellung erklïrt, eine auffassung die vielleicht auch müglich ist.

LUND, den 27. januar 1890. AXEL KOCK. 